Supporting Information for

\title{
Binding of Pertechnetate to Uranyl(VI) in Aqueous Solution. A Density-Functional Molecular Dynamics Study.
}

Michael Bühl ${ }^{*}$,Volodymyr Golubnychiy

\section{Details of Construction and Validation of the Tc Pseudopotential}

For technetium, a semicore (or small-core) pseudopotential was constructed following the procedure adopted previously for other transition-metal nuclei. ${ }^{1}$ A relativistic atomic reference calculation was performed for the $\left[1 s^{2} 2 s^{2} 2 p^{6} 3 s^{2} 3 p^{6} 3 d^{10}\right] 4 s^{2} 4 p^{6} 4 d^{5}$ state, and a pseudopotential was created for the core in brackets, using cutoff radii of 2.2, 2.2, and 2.0 a.u. for the $s$, $p$, and $d$ channels, respectively. ${ }^{2}$ Since this atomic calculation was performed for a $\mathrm{Tc}^{2+}$ state, the resulting pseudopotential is thus designed for technetium in high oxidation states.

Static optimizations and numeric frequency calculations were performed in the in the gas phase at the BLYP level, employing the CPMD program and the parameters detailed in reference 7 of the main paper (denoted CP-opt), and validated against experiment or SDD/6-31G* computations using Gaussian $03^{3}$ with the same functional (SDD denoting the Stuttgart-Dresden relativistic effective core potential, ECP, for Tc, ${ }^{4}$ see below for more details). Some representative results are summarized in Tables S1 and S2.

Table S1: Tc-O bond distance (in $\AA$ ) and harmonic vibrational frequencies (in $\mathrm{cm}^{-1}$ ) for $\mathrm{TcO}_{4}{ }^{-}(\mathrm{BLYP}$ level).

Parameter $\quad \mathrm{CP}_{-\mathrm{opt}^{a}}{ }^{a} \quad \mathrm{SDD} / 6-31 \mathrm{G}^{*} \quad$ Expt. [reference]

\begin{tabular}{llll}
\hline$r(\mathrm{Tc}-\mathrm{O})$ & 1.738 & 1.755 & $1.676[5]$ \\
$\mathrm{v}_{3}\left(T_{2}\right)$ & 880 & 887 & $912[6]$ \\
$\mathrm{v}_{1}\left(A_{1}\right)$ & 889 & 877 & 912 \\
$\mathrm{v}_{4}\left(T_{2}\right)$ & 308 & 321 & 325 \\
$\mathrm{v}_{2}(E)$ & 270 & 301 & - \\
\hline
\end{tabular}

${ }^{a}$ Box size $9.8692 \AA$ 
Table S2: Bond distance (in $\AA$ ), bond angles (in degrees) and relative energies (in $\mathrm{kcal} / \mathrm{mol}$ ) for cis- and trans $-\mathrm{TcO}_{2} \mathrm{~F}_{4}^{-}$(BLYP level).

\begin{tabular}{lllll} 
Complex & Parameter & CP-opt $^{a}$ & SDD/6-31G* & Expt. $^{b}$ \\
\hline cis $\left(C_{2 v}\right)$ & $r(\mathrm{Tc}-\mathrm{O})$ & 1.707 & 1.736 & $1.660(4)$ \\
& $r\left(\mathrm{Tc}-\mathrm{F}_{\mathrm{ax}}\right)$ & 1.917 & 1.926 & $1.876(3)$ \\
& $r\left(\mathrm{Tc}-\mathrm{F}_{\mathrm{eq}}\right)$ & 1.975 & 1.971 & $1.986(3)$ \\
& $a(\mathrm{O}-\mathrm{Tc}-\mathrm{O})$ & 101.3 & 102.2 & $102.5(3)$ \\
& $a\left(\mathrm{~F}_{\mathrm{ax}}-\mathrm{Tc}-\mathrm{F}_{\mathrm{ax}}\right)$ & 168.7 & 167.7 & $164.2(3)$ \\
& & & & \\
$\operatorname{trans}\left(D_{4 h}\right)$ & $r(\mathrm{Tc}-\mathrm{O})$ & 1.765 & 1.788 & - \\
& $r\left(\mathrm{Tc}-\mathrm{F}_{\mathrm{ax}}\right)$ & 1.910 & 1.922 & - \\
& $E_{\mathrm{rel}}{ }^{b}$ & 29.0 & 25.4 & - \\
& & & & \\
\hline
\end{tabular}

${ }^{a}$ Box size $13.67 \AA .{ }^{b}$ From reference $7 .{ }^{c}$ Relative to cis isomer.

By and large, the SDD/6-31G* results are reasonably well reproduced by the CP-opt data. No improvement could be brought about by choosing other cutoff radii for the $s, p, d$ channels during construction of the Tc pseudopotential. Compared to experiment, the BLYP functional displays the typical $^{8}$ over- $^{-}$ estimation of bond distances, which can be improved by using other functionals, e.g. BP86 (data not shown). The BLYP functional has been used because of its good performance to describe the properties of liquid water, ${ }^{9}$ as well as the dynamics, thermodynamic quantities, and reaction barriers of uranyl hydrate (see refs $6 \mathrm{c}$,d of the main paper). The use of hybrid functionals such as B3LYP, which is beneficial for many thermochemical applications, ${ }^{10}$ is prohibitively expensive in conjunction with CPMD.

\section{Details of Static Gas-Phase and PCM Computations}

Geometries have been optimized with the Gaussian 03 program $^{3}$ in the gas phase at the BLYP/LANL2DZ(U)/SDD(Tc)/6-31G** level, i.e. employing the Los Alamos relativistic ECP for U together with its $(3 \mathrm{~s} 3 \mathrm{p} 2 \mathrm{~d} 2 \mathrm{f})$ valence basis of contracted Gaussians, ${ }^{11}$ the Stuttgart-Dresden ECP for Tc, ${ }^{4}$ standard 6-31G(d,p) basis for all other elements, and a medium-sized integration grid (75 radial shells with 302 angular points per shell). Optimized coordinates are appended below. Very similar energetics were obtained when the small-core Stuttgart-Dresden ECP together with a [7s6p5d3f] valence basis set was used for $\mathrm{U}^{12}$ (where each of the most diffuse $s_{-}, p-, d$-, and $f$-primitives have been omitted from the 
original basis set, cf. the procedure adopted in reference 10), when relativistic all-electron calculations were performed employing the ZORA method (zeroth-order relativistic approximation) and a polarized triple-zeta basis of Slater functions as implemented in the ADF program package, ${ }^{13}$ or when the BP86 functional was used (data not shown).

Single-point energy computations were performed for the gas-phase geometries at the same DFT level using a polarizable continuum ${ }^{14}$ employing the parameters of water and UFF radii. For pristine nitrate and pertechnetate ions, the default settings in the Gaussian 03 program afford solvation energies of -61.6 $\mathrm{kcal} / \mathrm{mol}$ and $-55.4 \mathrm{kcal} / \mathrm{mol}$, respectively, which can be compared to experimental estimates of the free energies of solvation, $-71.7 \mathrm{kcal} / \mathrm{mol}$ and $-58.6 \mathrm{kcal} / \mathrm{mol}$, respectively. ${ }^{15}$ Apparently, the stronger hydration of $\mathrm{NO}_{3}{ }^{-}$vs. $\mathrm{TcO}_{4}{ }^{-}$is somewhat underestimated with the simple PCM approach.

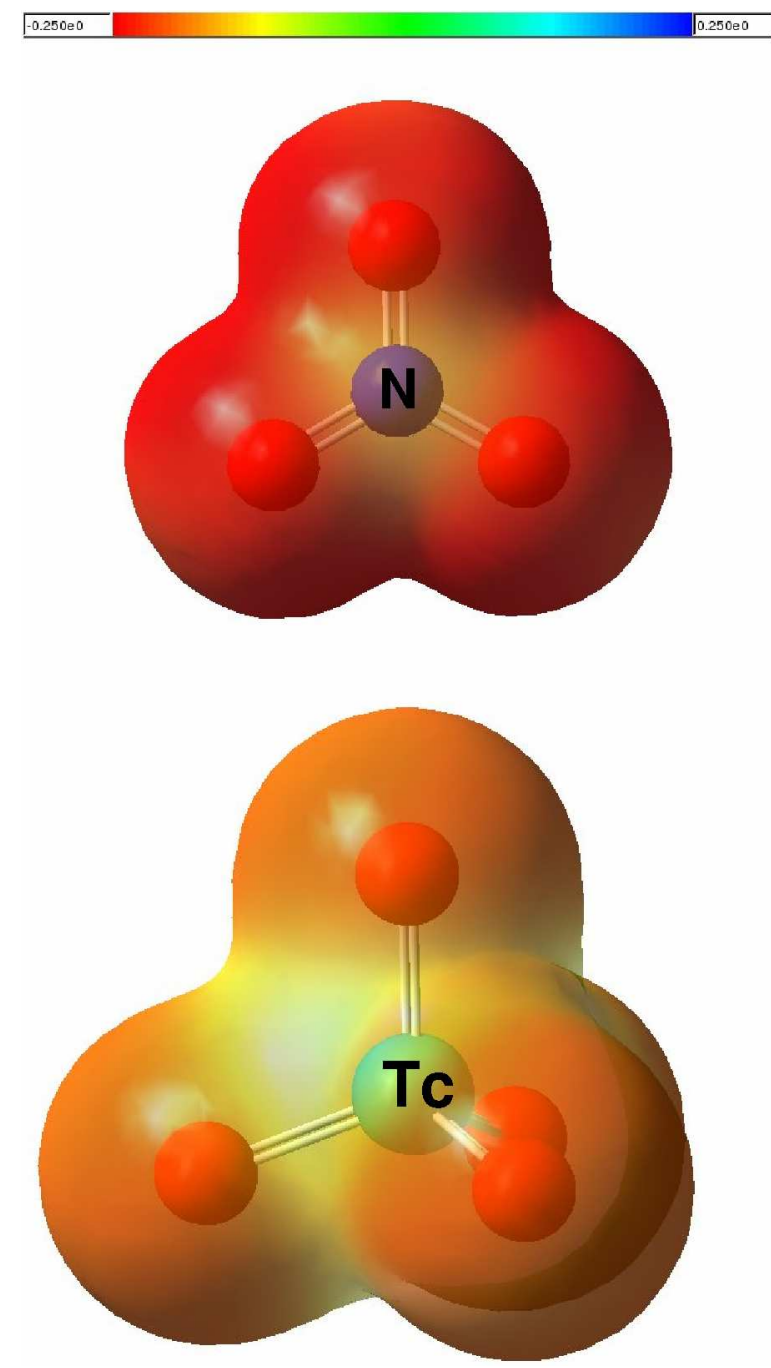

Figure S1: Electrostatic potential (BLYP/SDD(Tc)/6-31G* level), color-coded from -0.25 a.u. (red) to +0.25 a.u. (blue) and mapped on an isodensity surface $(\rho=0.008$ a.u.); top: nitrate, bottom: pertechnetate. 
The NPA ${ }^{16}$ and ESP computations for nitrate and pertechnetate have been performed at the BLYP/SDD(Tc)/6-31G* level detailed above. The approximate minima in the ESP values were located by inspecting the values on a suitable grid in space. The values reported in the main paper and plotted in Figure S1 were obtained in the gas phase for geometries fully optimized at that level, but very similar numbers are obtained with single-point PCM computations.

\section{Additional References}

1 For instance, for Mn: (a) M. Bühl, J. Phys. Chem. A. 2002, 106, 10505; for Co: (b) S. Grigoleit, M. Bühl, J. Chem. Theory Comput. 2005, 1, 181.

2 Using a program by D. R. Hamann based on the following references: (a) Hamann, D. R. Phys. Rev. B. 1989, 40, 2980; (b) Bachelet, G. B.; Hamann, D. R.; Schlüter, M. Phys. Rev. B 1982, 26, 4199; (c) Hamann, D. R.; Schlüter, M.; Chiang, C. Phys. Rev. Lett. 1979, 43, 1494.

3 M. J. Frisch, G. W. Trucks, H. B. Schlegel, G. E. Scuseria, M. A. Robb, J. R. Cheeseman, J. A. Montgomery, Jr., T. Vreven, K. N. Kudin, J. C. Burant, J. M. Millam, S. S. Iyengar, J. Tomasi, V. Barone, B. Mennucci, M. Cossi, G. Scalmani, N. Rega, G. A. Petersson, H. Nakatsuji, M. Hada, M. Ehara, K. Toyota, R. Fukuda, J. Hasegawa, M. Ishida, T. Nakajima, Y. Honda, O. Kitao, H. Nakai, M. Klene, X. Li, J. E. Knox, H. P. Hratchian, J. B. Cross, C. Adamo, J. Jaramillo, R. Gomperts, R. E. Stratmann, O. Yazyev, A. J. Austin, R. Cammi, C. Pomelli, J. W. Ochterski, P. Y. Ayala, K. Morokuma, G. A. Voth, P. Salvador, J. J. Dannenberg, V. G. Zakrzewski, S. Dapprich, A. D. Daniels, M. C. Strain, O. Farkas, D. K. Malick, A. D. Rabuck, K. Raghavachari, J. B. Foresman, J. V. Ortiz, Q. Cui, A. G. Baboul, S. Clifford, J. Cioslowski, B. B. Stefanov, G. Liu, A. Liashenko, P. Piskorz, I. Komaromi, R. L. Martin, D. J. Fox, T. Keith, M. A. Al-Laham, C. Y. Peng, A. Nanayakkara, M. Challacombe, P. M. W. Gill, B. Johnson, W. Chen, M. W. Wong, C. Gonzalez, and J. A. Pople, Gaussian 03, Gaussian, Inc., Pittsburgh PA, 2003.

4 Andrae, D.; Häußermann, U.; Dolg, M.; Stoll, H.; Preuß, H. Theor. Chim. Acta 1990, 77, 123.

5 Mean value, tetramethylammonium gegenion: G. E. German, M. S. Grigor'ev, A. F. Kuzina, V. I. Spitsin, Russ. J. Inorg. Chem. 1987, 32, 1089.

6 Raman fundamentals in aqueous solution, cited in: A. Müller, W. Rittner, Spectrochim. Acta 1967, $23 A, 1831$.

$7 \quad \mathrm{Li}^{+}$gegenion: W. J. Casteel, Jr., D. A. Dixon, N. LeBlond, H. P. A. Mercier, G. J. Schrobilgen, Inorg. Chem. 1998, 37, 340.

8 M. Bühl, H. Kabrede, J. Chem. Theory Comput. 2006, 2, 1282.

9 See for instance: a) M. Sprik, J. Hutter, M. Parrinello, J. Chem. Phys. 1996, 105, 1142; although potential shortcomings of this functional are now better appreciated, cf. b) J. VandeVondele, F. Mohamed, M. Krack, J. Hutter, M. Sprik, M. Parrinello, J. Chem. Phys. 2005, 122, 014515, and references cited therein. 
10 See e.g. for oxofluorides: Shamov, G. A.; Schreckenbach, G.; Vo, T. N. Chem. Eur. J. 2007, 13, 4932.

11 Ortiz, J. V.; Hay, P. J.; Martin, R. L. J. Am. Chem. Soc. 1992, 114, 2736, and references cited therein.

12 Küchle, W.; Dolg, M.; Stoll, H.; Preuss, H. J. Chem. Phys. 1994, 100, 7535.

13 (a) te Velde,G.; Bickelhaupt, F.M.; van Gisbergen, S.J.A.; Fonseca Guerra C.; Baerends, E.J.; Snijders, J.G.; Ziegler, T. J. Comput. Chem. 2001, 22, 931. (b) Fonseca Guerra, C.; Snijders, J.G.; te Velde, G.; Baerends, E.J. Theor. Chem. Acc. 1998, 99, 391. (c) Baerends, E.J.; Autschbach, J.; Bérces, A.; Bo, C.; Boerrigter, P.M.; Cavallo, L.; Chong, D.P.; Deng, L.; Dickson, R.M.; Ellis, D.E.; Fan, L.; Fischer, T.H.; Fonseca Guerra, C.; van Gisbergen, S.J.A.; Groeneveld, J.A.; Gritsenko, O.V.; Grüning, M.; Harris, F.E.; van den Hoek, P.; Jacobsen, H.; van Kessel, G.; Kootstra, F.; van Lenthe, E.; McCormack, D.A.; Osinga, V.P.; Patchkovskii, S.; Philipsen, P.H.T; Post, D.; Pye, C.C.; Ravenek, W.; Ros, P.; Schipper, P.R.T.; Schreckenbach, G.; Snijders, J.G.; Sola, M.; Swart, M.; Swerhone, D.; te Velde, G.; Vernooijs, P.; Versluis, L.; Visser, O.; van Wezenbeek, E.; Wiesenekker, G.; Wolff, S.K.; Woo, T.K.; Ziegler, T. ADF2004.01, SCM, Theoretical Chemistry, Vrije Universiteit, Amsterdam, The Netherlands..

14 PCM method as implemented in G 03: (a) Barone, V.; Cossi, M.; Tomasi, J. J. Comput. Chem. 1998, 19, 404-417; (b) Cossi, M.; Scalmani, G.; Rega, N.; Barone, V. J. Chem. Phys. 2002, 117, 43-54. c) Cossi, M.; Crescenzi, O. J. Chem. Phys. 2003, 19, 8863-8872.

15 As cited in: Marcus, Y. Biophys. Chem. 1994, 51, 111.

16 Reed, A. E.; Curtiss, L. A.; Weinhold, F. Chem. Rev. 1988, 88, 899-926

\section{Optimized Geometries}

Cartesian coordinates, BLYP/LANL2DZ(U)/SDD(Tc)/6-31G** optimized, together with total energies (E in a.u.) obtained at that level.

\begin{tabular}{|c|c|c|c|c|}
\hline \multirow{2}{*}{$\begin{array}{l}\text { Center } \\
\text { Number }\end{array}$} & \multirow{2}{*}{$\begin{array}{l}\text { Atomic } \\
\text { Number }\end{array}$} & \multicolumn{3}{|c|}{ Coordinates (Angstroms) } \\
\hline & & $\mathrm{X}$ & $\mathrm{Y}$ & Z \\
\hline 1 & 7 & 0.000000 & 0.000000 & 0.000000 \\
\hline 2 & 8 & 0.000000 & 1.286547 & 0.000000 \\
\hline 3 & 8 & -1.114182 & -0.643273 & 0.000000 \\
\hline 4 & 8 & 1.114182 & -0.643273 & 0.000000 \\
\hline \multicolumn{5}{|c|}{$\mathrm{TcO} 4-\mathrm{Td} \quad(\mathrm{E}=-381.86261)$} \\
\hline 1 & 43 & 0.000000 & 0.000000 & 0.000000 \\
\hline 2 & 8 & 1.013502 & 1.013502 & 1.013502 \\
\hline 3 & 8 & -1.013502 & -1.013502 & 1.013502 \\
\hline 4 & 8 & -1.013502 & 1.013502 & -1.013502 \\
\hline 5 & 8 & 1.013502 & -1.013502 & -1.013502 \\
\hline
\end{tabular}


$\mathrm{H} 2 \mathrm{O} \mathrm{C} 2 \mathrm{~V} \quad(\mathrm{E}=-76.39687)$

\begin{tabular}{|c|c|c|c|c|}
\hline 1 & 8 & 0.000000 & 0.000000 & 0.121556 \\
\hline 2 & 1 & 0.000000 & 0.763584 & -0.486224 \\
\hline 3 & 1 & 0.000000 & -0.763584 & -0.486224 \\
\hline [ UO2 & $1-\mathrm{NC}$ & $(E=-787$ & 384931 & \\
\hline 1 & 92 & -0.452726 & 0.013957 & -0.055200 \\
\hline 2 & 8 & -1.980730 & -1.975588 & 0.547107 \\
\hline 3 & 8 & 3.767398 & 0.944785 & 0.535155 \\
\hline 4 & 8 & -0.086134 & 2.534755 & -0.457417 \\
\hline 5 & 8 & 1.775815 & 0.547926 & -0.367503 \\
\hline 6 & 8 & -2.587181 & 1.148209 & 0.781686 \\
\hline 7 & 8 & -0.039563 & -0.107534 & 1.706280 \\
\hline 8 & 8 & 3.102487 & -1.152683 & 0.251284 \\
\hline 9 & 8 & -1.207072 & -0.006911 & -1.707437 \\
\hline 10 & 8 & 0.780393 & -2.060883 & -0.754947 \\
\hline 11 & 7 & 2.962641 & 0.095925 & 0.185515 \\
\hline 12 & 1 & -1.777264 & -2.476171 & 1.361926 \\
\hline 13 & 1 & -2.245380 & -2.640269 & -0.118521 \\
\hline 14 & 1 & 0.870491 & 2.742115 & -0.484681 \\
\hline 15 & 1 & -0.511338 & 3.069211 & -1.155512 \\
\hline 16 & 1 & -2.758222 & 1.343875 & 1.723139 \\
\hline 17 & 1 & -3.438067 & 1.243339 & 0.312737 \\
\hline 18 & 1 & 0.844674 & -2.291188 & -1.703084 \\
\hline 19 & 1 & 1.724094 & -1.923018 & -0.429860 \\
\hline
\end{tabular}

$[\mathrm{UO} 2(\mathrm{H} 2 \mathrm{O}) 3(\mathrm{~h} 2-\mathrm{NO} 3)]+\mathrm{C} 2 \mathrm{~V} \quad(\mathrm{E}=-711.46674)$

\begin{tabular}{|c|c|c|c|c|}
\hline 1 & 92 & 0.000000 & 0.000000 & 0.337401 \\
\hline 2 & 8 & 0.000000 & 0.000000 & -3.834008 \\
\hline 3 & 8 & 0.000000 & 1.094329 & -1.891383 \\
\hline 4 & 8 & -1.808083 & 0.000000 & 0.453413 \\
\hline 5 & 8 & 0.000000 & 2.536057 & 0.604026 \\
\hline 6 & 8 & 0.000000 & -2.536057 & 0.604026 \\
\hline 7 & 8 & 0.000000 & -1.094329 & -1.891383 \\
\hline 8 & 8 & 1.808083 & 0.000000 & 0.453413 \\
\hline 9 & 8 & 0.000000 & 0.000000 & 2.880475 \\
\hline 10 & 7 & 0.000000 & 0.000000 & -2.621048 \\
\hline 11 & 1 & -0.781228 & 3.062322 & 0.338715 \\
\hline 12 & 1 & 0.781228 & -3.062322 & 0.338715 \\
\hline 13 & 1 & -0.781228 & -3.062322 & 0.338715 \\
\hline 14 & 1 & 0.781228 & 3.062322 & 0.338715 \\
\hline 15 & 1 & 0.785576 & 0.000000 & 3.461477 \\
\hline 16 & 1 & -0.785576 & 0.000000 & 3.461477 \\
\hline
\end{tabular}




$\begin{array}{rrrrr}{[\mathrm{UO} 2(\mathrm{H} 2 \mathrm{O})} & 4(\mathrm{~h} 1-\mathrm{TCO} 4)]+\mathrm{C} 1 & (\mathrm{E}=-889.38826) & \\ -1 & 92 & 1.199135 & -0.061043 & -0.032534 \\ 2 & 43 & -2.706772 & -0.083520 & 0.067349 \\ 3 & 8 & 0.996331 & -0.197955 & 1.761039 \\ 4 & 8 & 1.767720 & 0.012391 & -1.754390 \\ 5 & 8 & 2.059278 & 2.268814 & 0.691251 \\ 6 & 8 & 1.318769 & -2.590939 & -0.298606 \\ 7 & 8 & 3.674637 & -0.451636 & 0.398858 \\ 8 & 8 & -0.089189 & 2.078819 & -0.924796 \\ 9 & 8 & -0.971709 & -0.653453 & -0.235220 \\ 10 & 8 & -3.116839 & -0.301396 & 1.721457 \\ 11 & 8 & -3.799968 & -0.954435 & -0.929311 \\ 12 & 8 & -2.710150 & 1.617590 & -0.335899 \\ 13 & 1 & -1.064781 & 2.139185 & -0.707976 \\ 14 & 1 & -0.031625 & 2.161337 & -1.898644 \\ 15 & 1 & 1.472750 & 2.915903 & 0.241737 \\ 16 & 1 & 2.059457 & 2.503971 & 1.640276 \\ 17 & 1 & 0.776054 & -3.172982 & 0.269946 \\ 18 & 1 & 1.335880 & -3.007831 & -1.182801 \\ 19 & 1 & 4.119028 & -0.573749 & 1.259676 \\ 20 & 1 & 4.372953 & -0.380904 & -0.280143 \\ -------------14\end{array}$

\begin{tabular}{|c|c|c|c|c|}
\hline 1 & 92 & 0.000000 & 0.000000 & 0.961759 \\
\hline 2 & 8 & 0.000000 & 1.800176 & 1.112616 \\
\hline 3 & 8 & 2.504478 & 0.000000 & 1.487243 \\
\hline 4 & 8 & -2.504478 & 0.000000 & 1.487243 \\
\hline 5 & 8 & 0.000000 & -1.800176 & 1.112616 \\
\hline 6 & 8 & 0.000000 & 0.000000 & 3.493003 \\
\hline 7 & 43 & 0.000000 & 0.000000 & -2.334533 \\
\hline 8 & 1 & 3.027790 & 0.780027 & 1.211359 \\
\hline 9 & 1 & -3.027790 & -0.780027 & 1.211359 \\
\hline 10 & 1 & -3.027790 & 0.780027 & 1.211359 \\
\hline 11 & 1 & 3.027790 & -0.780027 & 1.211359 \\
\hline 12 & 1 & 0.000000 & -0.786865 & 4.071982 \\
\hline 13 & 1 & 0.000000 & 0.786865 & 4.071982 \\
\hline 14 & 8 & 1.323336 & 0.000000 & -1.107864 \\
\hline 15 & 8 & -1.323336 & 0.000000 & -1.107864 \\
\hline 16 & 8 & 0.000000 & 1.411244 & -3.306389 \\
\hline 17 & 8 & 0.000000 & -1.411244 & -3.306389 \\
\hline
\end{tabular}

